\title{
Scène de crime dans le roman policier : essai d'analyse lexico-syntaxique
}

\author{
Teresa Muryn, Malgorzata Niziolek, Alicja Hajok, Wojciech Prazuch, Katarzyna Gabrysiak \\ Université Pédagogique de Cracovie (Pologne) \\ teresa.muryn@gmail.com,mniziolek1@gmail.com, alicjahajok@gmail.com, \\ prazuch.wojciech@gmail.com, gabrysiak.katarzyna81@gmail.com
}

\begin{abstract}
Résumé. Le présent article a pour but d'exposer l'analyse des structures rhétorico-lexicosyntaxiques propres au roman policier visant à établir la matrice lexico-syntaxique du genre en question. Le choix du roman policier résulte du fait que sa structure interne est stable indépendamment de l'auteur, de l'époque ou d'autres facteurs extérieurs. Ladite analyse, qui repose sur un corpus rassemblant des romans policiers en français et comptant actuellement 35850957 tokens, se situe sur deux niveaux : le premier concerne l'histoire elle-même, le second constitue l'échafaudage textuel de cette histoire. Elle consiste en extraction des structures RLS pertinentes pour les scènes normatives du genre; dans le cas du présent article pour la scène normative descriptive de la scène de crime dans le roman policier.
\end{abstract}

\begin{abstract}
The purpose of this article is to analyse the rhetorical, lexical and syntactic (RLS) structures which are typical of crime novels. In a further perspective, the conducted research will make it possible to distinguish the lexical and syntactic matrix of the genre. The choice of crime novels results from the existence of a constant, internal structure, which is independent of the author, the period or other criteria. The proposed analysis is based on the corpus of crime novels in the French language, which currently includes 35850957 tokens. The research consists in the extraction of the RLS structures which are characteristic of the normative scenes of the genre, and, in the case of this article, of the normative descriptive scene - the scene of crime.
\end{abstract}

\section{Remarques préliminaires}

Notre objectif est de proposer une analyse innovante applicable aux textes littéraires. Nous tenterons de démontrer dans ce qui suit qu'il est possible d'extraire des structures rhétorico-sémantico-syntaxiques en vue de proposer une matrice du genre.

Les recherches récentes en sémantique lexicale et en phraséologie exploitant les méthodes et les moyens de la linguistique de corpus ont démontré que les approches traditionnelles et leurs délimitations d’unités lexicales s'avèrent insuffisantes. En général, le phénomène phraséologique trouve son expression dans toutes sortes de manifestations.

Les structures plus ou moins figées, préconstruites ou semi-préconstruites ont fait l'objet de plusieurs études : unités phraséologiques ou phraséologies, phrases semi-préconstruites (Sinclair, 1991), collocations (Halliday, 1961 ; Blumenthal, Tutin), cadres collocationnels (Renouf \& Sinclair, 1991), expressions figées (Gross, 1996), séquences figées (Mejri, 1997), colligations (de type lexical ou textuel Hoey, 2005), segments répétés (Salem, 1986), motifs (Longrée \& Mellet, 2012 ; Grossmann, 2015), unités lexicales étendues (Sinclair, 2004), séquences ou patrons formulaires (Biber 2009), routines discursives (Née, Sitri, Veniard, 2014), matrices lexicales (Anscombre, 2011), patrons (Hunston \& Francis, 2000), constructions (Fillmore, 1988 ; Bouveret \& Legallois 2012 pour le français), constructions 
préformées (Schmale, 2013). Le point commun de ces dénominations est le postulat de l'existence de structures plus ou moins figées.

\section{Un texte littéraire - Le roman policier}

Le texte littéraire, considéré comme stylistiquement imprévisible, est souvent exclu des analyses linguistiques[1]. Mais, depuis quelques années, on observe un intérêt croissant pour les textes littéraires (cf. Legallois, Siepmann, Beauvisage).

L’idée de travailler sur le roman policier comme genre est née des analyses réalisées à partir d'un corpus restreint de textes de Georges Simenon. Les premiers résultats nous ont incités à élargir notre corpus et à nous pencher sur le roman policier. Parler du roman policier présuppose bien sûr qu'un tel objet existe et qu’il possède une cohérence interne. Le roman policier, grâce à sa structure narrative plus ou moins stable (et abondamment décrite, entre autres par Van Dine (1928), Todorov (1971), Lasic, (1972), Lits (1993)) constitue un type de texte particulièrement propice pour y chercher des structures langagières qui créent des effets de genre.

Le roman policier est construit sur un schéma qui est d'ailleurs très bien décrit par de nombreux chercheurs. Cependant leurs descriptions concernent les traits dominants au niveau de l'histoire. Van Dine publie en 1928, dans « L'American magazine », les 20 règles du roman policier (Lits, 1993). Mais c'est dans les années 1960 et 1970 que le roman policier est devenu un objet d'études approfondies. Les structuralistes, en particulier Barthes, Todorov, Greimas, Brémond, ont essayé de créer une grammaire universelle de la littérature. La première étape dans la réalisation de cet objectif était de créer un modèle de production pour la narration. Dans sa Poétique de la prose, Todorov consacre un chapitre à la « Typologie du roman policier » (1971). Parmi les études récentes consacrées au roman policier, il faut noter le texte de Marion François dans lequel l'auteur présente le cliché comme élément constitutif du genre (2009) :

[...] parmi les signes de reconnaissance, les clichés, pris en tant qu'« associations figées du lexique » (Jean-Louis Dufays, Stéréotype et lecture, Liège, Mardaga, 1994, p. 81) semblent justement fondamentaux, là où d'autres critères de définition, axés sur d'autres stéréotypies, échouent à couvrir une production mouvante et variée, dès l’origine du « genre » : ceux qui supposent une structure fixe (question/réponse), des personnages immuables (enquêteur/criminel/témoins), un processus récurrent (l'enquête).

On peut déduire de ces travaux que l'élément commun des textes policiers, c'est l'existence d’une cohérence interne, d'une matrice adaptable[2] qui permet de décrire leur structure fonctionnelle et organisationnelle. Cette structure complexe s’extériorise à travers le langage.

Comme nous l'avons déjà dit, plusieurs études qui ont été consacrées au roman policier se focalisent au niveau de l'histoire. Les schémas, proposés entre autres par les structuralistes, décrivent différentes fonctions du roman policier. Cependant les critères formels (fondés sur l'observation de la structure langagière) définitoires du genre policier restent à définir. Thomas Beauvisage tente une modélisation de ce qu'est le genre policier à partir des critères purement statistiques (2001)[3].

\subsection{Roman policier - discours ou genre ?}

François Rastier et Denise Malrieu (2000) distinguent quatre niveaux hiérarchiques supérieurs d'analyse : les discours (p. ex. juridique, littéraire, scientifique), les champs génériques (dans le cadre du discours littéraire : poésie, genre narratif, théâtre), les genres proprement dits (p. ex. la comédie, le « roman sérieux ", le roman policier), les sous-genres (ex. roman par lettres). S’appuyant sur cette définition et sur ces constats Beauvisage (2001) remarque que la qualification d'« interactions normées » rappelle que les genres ont un caractère normatif [...] que pour un champ d'activité donné, différents genres sont proposés au locuteur, qui sont plus ou moins prescriptifs selon l'activité [...]. C'est [...] aspect de variation possible [...] amène à envisager nécessairement l'étude des genres dans la perspective d'une linguistique de corpus, 
seule à même de détecter les constantes et les variations au sein d'un genre donné, et d'identifier les éléments fondamentaux de l'écriture pour ce genre ». L'auteur propose de " prendre en compte le genre comme une donnée, mais en même temps [de] tenter de le caractériser et de lui donner une unité qui ne soit plus liée à la situation ou à l'intuition, mais qui soit proprement textuelle »[4].

Dans notre analyse, nous adaptons, après les auteurs cités, la définition du genre et, par conséquent, nous identifions le roman policier comme un type de genre.

\subsection{Corpus de travail}

Le corpus sur lequel se fonde cette étude se compose de romans policiers en français venant d'auteurs reconnus, aussi bien français qu'étrangers : Gaston Leroux, Maurice Leblanc, Conan Doyle, Georges Simenon, Fred Vargas, Agatha Christie, Jean-Christophe Grange, Arnaldur Indridason, Maxime Chattam, Dorothy L. Sayers, Harlam Coben, Jean-Claude Izzo, Pierre Magnan, Boileau-Narcejac, Exbrayat, et d’autres. Il se compose actuellement de 35850957 tokens et s'enrichit progressivement[5].

Ce n'est pas par hasard que nous y avons réuni des textes d'auteurs français et étrangers traduits en français. Nous n'avons pas non plus respecté l’homogénéité temporelle des éditions - un siècle sépare Arsène Lupin du commissaire Adamsberg (Fred Vargas). Une telle décision a été dictée par le postulat de l'existence d'une matrice universelle du genre étudié indépendamment de l'époque et du lieu de production.

Quant a la traduction, pendant l'analyse des relations existant entre deux textes, celui de départ et celui d'arrivée, les composantes des couples de segments doivent partager une série de caractéristiques permettant d'identifier l'un comme la contrepartie de l'autre. Nous nous référons ici à la notion d'invariance (cf. : Reiss, Kade) définie par la branche théorique de la traductologie. Il va sans dire que les choix traductionnels peuvent se faire au détriment de certains éléments du texte. Cependant, en dépit des écarts auxquels tout traducteur doit se résoudre, il doit identifier le type de texte et respecter un certain ordre des priorités qui en sont tributaires. Être fidèle à l'original signifie dans ce cas-là assurer l'invariance de certains schémas lexico-syntaxiques appropriés qui discriminent un type de texte, par exemple un roman policier, tel un code ADN. On ne pourrait donc faire l'impasse sur certains éléments car ils représentent les marques obligatoires du genre. L'évolution du genre peut modifier certaines caractéristiques, mais le schéma qui permet de reconnaître un roman policier comme tel doit rester stable (ce type de texte est régi par des principes organisateurs communs à l’ensemble des textes policiers).

\section{Méthodologie du travail}

Nous partons du principe que la répétition lexicale assure au texte sa cohésion. De plus, la prise en compte de cette répétition rend possible l'observation du mode d'organisation du texte. En reprenant les propos de Legallois (2006) portant sur les réseaux phrastiques basés sur l'identification de phrases ayant au moins trois lexèmes en commun - ce qui « permet de constater [...] qu'un mode d'organisation réticulaire est à l'œuvre dans certains types de textes » - et en nous appuyant par ailleurs sur sa vision de la répétition définie comme " une relation particulière de cohésion, un phénomène par lequel dans un texte donné 1) une unité lexicale est reprise sous la même forme ou sous une forme dérivée ; 2) un contenu informationnel est repris en totalité ou partiellement par une unité sémantique apparentée [...] une ellipse, par une anaphore » (Legallois, 2006 : 57), nous pouvons dire que les unités réticulaires ne portent pas seulement sur les éléments lexicaux, mais sur toutes les structures rhétorico-lexicosyntaxiques et la répétition de ces structures ne se fait pas au niveau d'un texte mais au niveau d'un genre.

Cette analyse s'appuie sur notre vision du rapport entre la phrase et le discours, qui réoriente l'ascendance classique allant du simple au complexe. Dans les travaux de notre groupe, nous partons du constat que chaque type de discours se caractérise par l'organisation de structures sémantiques complexes qui y dominent, en même temps que par le choix de prédicats et d'arguments, la spécification de positions impliquées, etc. Les réalisations lexico-syntaxiques de ces structures sont propres à un discours donné. 
Une structure sémantique complexe peut ainsi revêtir la forme d'une phrase complexe, d'une phrase simple, d'un SN, etc., ou bien être inférée en totalité ou en partie. La recherche de séquences lexicosyntaxiques parallèles réalisant une même structure sémantique dans un type de discours à l'aide de moyens offerts par la linguistique de corpus et exploitant des méthodologies offertes par la phraséologie dite étendue (Sinclair, 2004 ; Tutin \& Legallois, 2013) permettra d'extraire le schéma rhétoricosyntaxico-lexical d'un type de discours en éliminant en même temps le problème de la polysémie, de l’inférence et de l'ambiguïté. Elle permettra surtout de reconnaître et de créer différents genres selon des critères linguistiques et non pas intuitifs. Dans la suite de ce travail, nous utilisons les dénominations suivantes :

\subsection{Motif sémantique}

Le motif sémantique est une représentation abstraite de toutes les structures réalisant le même modèle prédicat-argument. Il se réalise à travers la structure lexico-syntaxique (voir infra). Notre définition du motif se distingue des autres définitions du terme. Nous situons celui-ci sur le plan sémantique, en lui assignant une représentation encore plus abstraite que celles qui ont été proposées jusqu'à présent. Longrée et Mellet (2013 : 66) définissent le motif comme : " un cadre collocationnel' accueillant un ensemble d'éléments fixes et de variables, susceptible d'accompagner la structuration textuelle et simultanément, de caractériser des textes de genres divers, voire de permettre la détection, au sein d'un même texte, des passages de registres différents ».

Le motif est aussi une notion issue d'une conception élargie de la phraséologie (Legallois \& Tutin, 2013). Les critères de fréquence, la récurrence, la mémorisation entrent dans la définition des motifs (Longrée \& Mellet, 2013 : 66). Grossmann étend la définition du motif : « Le recours à la notion de motif plutôt qu'à celle de frame prend en compte le fait qu'on se situe dans un modèle sémantique et textuel plutôt que cognitif : on vise une représentation abstraite de stéréotypes textuels » (Grossmann, 2015 : 40).

\subsection{Structure lexico-syntaxique}

Il s’agira de toute réalisation du motif sémantique grammaticalement complète (désormais - structure LS). Sur ce point, on peut voir l’outil SDMC (Séquential Data Mining under Constraints) développé par le laboratoire Greyc : https://sdmc.greyc.fr qui permet de dégager des constructions lexico-syntaxiques, par exemple celles retenues dans les romans d'amour Harlequin : V-PS le NCCorps sur DET POSS NCCorps - Il appliqua la bouche sur seins, avec douceur, d'abord, puis avec force (Thierry Charnois, LIPN, Fouille de motifs pour le traitement automatique des langues, séminaire externe du 13 novembre 2015 à Université Stendhal-Grenoble 3, Grenoble) ou encore celles identifiées dans le discours idéologique, tel le motif exprimant la dimension hétéroglossique : ADJ NC contre le NC - C’est un long pamphlet contre les juifs (Sophie Anquetil, CeReS, Université de Limoges, Dominique Legallois, CRISCO, Université de Caen, La phraséologie de l'idéologie, Europhrase, 2014). Cependant, d’après nous, il serait intéressant de pouvoir associer ces structures lexico-syntaxiques aux genres.

Certaines de ces structures LS contiennent un élément collocationnel. Les collocations se trouvent à la frontière de la combinatoire libre et de la combinatoire figée. Certaines collocations ne peuvent être retrouvées que dans un genre donné. Elles se définissent comme « une cooccurrence conventionnelle, résultant d'une forte contrainte sémantique de sélection qui se manifeste dans la valence d'une unité lexicale, et qui a pour effet de restreindre la compatibilité des mots avec l'unité en question [...]. On notera que, contrairement aux locutions, les collocations ne sont pas des cas de figement, puisque les assemblages lexicaux restent libres » (Neveu, 2004 : 70)[6].

\section{Matrice englobante du roman policier et ses scènes normatives}

Dans nos recherches, nous postulons l'existence d'une matrice englobante du roman policier qu'on définit comme l'ensemble des structures LS associées aux scènes normatives (désormais RLS). La question qui 
se pose est de savoir comment, avec quels principes méthodologiques, découvrir et délimiter les séquences que l'on pourrait qualifier d'appropriées à un genre, comment trouver cette matrice lexicosyntaxique du genre dont nous postulons l'existence et qui serait adoptée par tous les représentants du genre. La méthode purement inductive basée sur l'extraction d'unités à haute fréquence ne semble pas suffisante. Certaines unités lexicales ayant un caractère qui semble normatif tels que « corps », « cadavre ", " dépouille», " mort ", etc. n’ont pas de fréquence élevée dans le roman policier et c'est bien compréhensible. Ce qui les caractérise, c'est leur apparition dans tous les romans policiers, le plus souvent dans la description d'une scène de crime. C'est pour cette raison qu'il est intéressant de compléter l'analyse par une méthode déductive permettant la décomposition du texte en unités textuelles plus petites, une sorte de module-moule, que nous avons appelées scènes normatives. Ces scènes normatives à caractère thématique construisent le modèle complet du genre spécifique que nous appelons matrice englobante. Il nous semble nécessaire de distinguer entre deux types de scènes normatives, auxquelles nous attribuons une terminologie de travail, à savoir : scènes normatives descriptives et scènes normatives opérationnelles. Prenons comme exemple la scène de crime vue comme scène normative descriptive. Pour l'identifier comme telle, elle exige les éléments suivants (structures RLS) : la découverte du corps, la description du corps, la description des lieux, les traces du crime. Le niveau opérationnel, vu comme scène normative opérationnelle, qui ne constitue pas l'objet de cette étude, s'exprime à travers différentes énonciations et propos routiniers utilisés dans les situations stéréotypées du roman policier. Chaque scène normative se définit par un ensemble obligatoire de structures RLS.

Nous voulons vérifier ce postulat théorique sur le roman policier, genre littéraire relativement stable, ayant ses propres caractéristiques normatives[7]. Le but du projet que nous réalisons est de déterminer quelles structures lexico-syntaxiques y prédominent, pour établir finalement la matrice que tous les romans policiers adaptent.

Bien sûr, dans nos analyses, nous nous sommes servis des descriptions de discours qui avaient dans leurs principes une décomposition du texte global (ici le genre du roman policier) en sous-structures. Celle qui nous a inspirés le plus est une approche cognitive qui propose une architecture du texte composée de modules enchaînés et hiérarchisés illustrant une situation standardisée que le texte analysé représente. Autrement dit, nous reconnaissons dans un texte le type de situation pour lequel l'expérience commune prévoit certains comportements comme obligatoires. Les modules en question étiquettent ces comportements et les mettent dans un ordre précis. Dans les théories cognitives, surtout celles de frame et du script, l'ensemble de ces connaissances stockées dans la mémoire constitue un cadre. La notion de cadre, autrement dit de frame ou de schéma, a été introduite à la psychologie par Bartlett (1932), et ensuite développée par plusieurs auteurs, entre autres par Minsky (1975) Schank \& Abelson (1977), Denhière \& Baudet (1992), Ehrlich (1994), Kintsch (1998), Van Oostendorp \& Goldman (1999), López Alonso \& Séré (2001), Van Dijk (2008). En général, le cadre est un schéma mental dont chaque individu dispose et grâce auquel il garde en mémoire des connaissances permettant d'organiser toutes les informations acquises au cours du processus de perception des objets, des situations, des événements. Ces schémas permettent de reconnaître des concepts déjà assimilés ainsi que de traiter et de comprendre de nouvelles informations.

Le roman policier se soumet à une telle structure sous-jacente qui lui assure l'appartenance à ce genre. Comme il représente une réalité en elle-même fortement standardisée, l'enchaînement de modules textuels représentant des situations dans l'ordre n'est pas étonnant. Bien au contraire : la reconnaissance de l'architecture modulaire, p. ex. celle du genre, assure la reconstruction de la structure globale, même si l'enchaînement des modules-constituants a été bouleversé.

Pourtant, ni l'identification ni l'enchaînement des modules ne sont dictés, dans ces analyses, par une analyse textuelle - ils le sont par l'expérience extralinguistique des codeurs et des décodeurs de textes (auteurs et lecteurs) qui identifient une histoire décrite à une histoire vécue. Par conséquent, les analyses cognitives se rapprochent des analyses littéraires du genre en question (surtout celles datant des années 1970) qui ont proposé aussi, sans le nommer, des modules obligatoires pour le genre. 
Les analyses cognitives, surtout du frame et du script, comme celles des structuralistes, n'ont pas pris en compte le texte global du roman policier mais l'histoire qu'il décrit. C'est pour cette raison que tout ce qui appartient au cadrage métadiscursif du texte échappe à l’analyse. Pourtant, une analyse détaillée, un dépouillement du corpus homogène des romans policiers, a révélé l'existence du métadiscours approprié dans le genre analysé. Nous reviendrons au problème un peu plus tard.

La plupart des études se donnant pour but une analyse phraséologique du discours littéraire se concentrent sur la recherche des séquences statistiquement marquées. Tout en acceptant les buts et les résultats de ces recherches, nous ne pouvons pas nous empêcher de faire une remarque : elles permettent de trouver seulement des séquences de mots privilégiées et non pas des structures lexico-syntaxiques complètes privilégiées. Or, le but que nous nous donnons dépasse le cadre d’une analyse phraséologique standard. Ce qui nous intéresse ce sont des structures ayant leurs sélection et réalisation spécifiques dans un genre parce qu'elles seraient déterminées par le genre en question. Autrement dit, tel genre - telles structures. Mais le problème est plus complexe. Nous voulons savoir quel est le rôle des séquences sélectionnées dans la structure globale, quelle scène normative (module-moule) elles sont en train de saturer et si l'on peut établir cette régularité pour un genre.

D’après nous, pour répondre à ces questions, il faut dépasser le cadre d'une description lexico-syntaxique pure et étendre l'analyse au niveau sémantique où l'on pourrait trouver de vrais motifs sémantiques qui se réalisent à travers plusieurs constructions formelles plus ou moins fréquentes. On cherchera donc des structures sémantiques obligatoires pour une scène obligatoire et ses réalisations les plus fréquentes.

Cette optique méthodologique et une analyse phraséologique appliquée au corpus ont déjà permis de dégager, dans les textes appartenant au genre du policier, deux niveaux d'analyse : celui de l'histoire et celui de l'échafaudage textuel de cette histoire fonctionnant comme marquage métadiscursif. L'histoire consiste en description de faits tandis que le marquage métadiscursif opère entre l'auteur du texte et son lecteur, introduit, conclut, commente ou fait avancer l'histoire. Toutefois, il est nécessaire de s'appuyer sur le modèle cognitif pour extraire des concepts-clés obligatoirement présents dans les scènes normatives. Ce sont justement ces concepts qui donnent accès à des structures RLS sélectionnées par les scènes normatives du genre. Elles participent alors à la création de la matrice du roman. Étant donné qu'elles peuvent se manifester sous différentes variantes formelles, il semble nécessaire de les classer sous un motif sémantique propre à toutes les variantes.

\section{Dégagement des structures RLS dans une scène normative: scène de crime}

L'objectif de cette partie est de proposer une description de la scène normative - en l'occurrence, la scène de crime. Cette description est basée sur nos connaissances extralinguistiques et aussi sur les observations linguistiques de notre corpus.

Nos connaissances extralinguistiques nous permettent de dégager toujours les mêmes éléments constituant une scène de crime, c'est - à - dire la découverte et la description du cadavre, la description des lieux, la description de la mise en place des techniques policières, les mêmes protagonistes (le commissaire, la police technique et scientifique, le médecin légiste, les témoins), les mêmes procédures à suivre. L’introduction de tous ces éléments dans un roman policier est relativement répétitive et permet d'extraire des structures RLS constituant finalement toute la scène normative.

Dans les études phraséologiques des textes, l’attention est portée aux collocations au sens traditionnel du terme. Elles permettent de dégager des constructions polylexicales autour de la base collocationnelle. Ces constructions peuvent apparaître presque dans tous les genres parce qu'elles ont une valeur lexicale stable. Elles font déjà partie de différents dictionnaires de collocations ou de combinaison de mots. Cependant, ce qui est spécifique pour un genre textuel c'est que ces collocations sont intégrées dans les RLS. Dans notre approche elles sont donc considérées comme des constructions complètes préfabriquées. Par exemple : [RETROUVER+MORT] + défiguré; empoisonnés ; d'une hémorragie interne ; frappé à la poitrine par une décharge de fusil à pompe calibre 12 ; plus précisément assassinée, d'une balle dans la 
tête. Elles sont toutes construites à l'aide des mêmes éléments variables : LIEU, TEMPS, la cause de la mort. Même si un de ces éléments n'apparaît pas à la surface de la phrase, il est tout à fait possible de l'intégrer dans le RLS par exemple en reformulant On les a retrouvés morts, empoisonnés on obtient On les a retrouvés morts, empoisonnés à huit heures du matin chez eux, mais aussi On l'a retrouvée morté, pendue.

Pour illustrer cette thèse, nous proposons quelques exemples de ces RLS dégagées dans les scènes de crime disponibles dans notre corpus. Nous les avons divisées en groupes. Chaque groupe possède une étiquette sémantique, quelques exemples authentiques relevés du corpus et, à la fin, un tableau récapitulant des entrées commutantes. La liste des entrées n’est pas complète. Elle ne contient que des éléments issus de quelques exemples que nous avons sélectionnés arbitrairement de notre corpus pour présenter la méthode.

\subsection{Mesures conservatoires et état des lieux}

- Vous voulez voir la place où il est mort !... C’est curieux... Cependant ce ne sont que des policiers de Nevers qui ont fait ça... Quand ils ont emporté le corps, ils ont d'abord dessiné son contour sur le plancher, avec de la craie... Attention de ne toucher à rien, hein !...

- Relever les empreintes, prendre des photos du corps.

- Le médecin légiste examinait le cadavre et les photographes installaient leurs appareils

- Au dehors, le commissaire, le nez au sol, examina à nouveau le sol fangeux et gelé où le corps s'était écrasé.

- Le labyrinthe. - Je peux descendre ? \{S\}On lui passa des sur chaussures et une charlotte de papier. \{S\}Elle emprunta l'escalier de fer qui permettait de plonger dans la fosse.\{S\} Les techniciens de l'Identité judiciaire s'écartèrent. $\{S\}$ Elle s'accroupit, examina la zone qui l'intéressait : cette tête monstrueuse d'animal enchâssée sur un corps d'homme. \{S\}La deuxième option était la bonne.\{S\} La tête avait été enfoncée a pleines forces sur celle de la victime. $\{S\}$ Au-dessous, le crâne devait être en bouillie. - À mon avis, il a creusé l'intérieur du cou de la bête. $\{S\}$ Anaïs se retourna vers celui qui venait de parler.\{S\} Michel $\{S\} L$ Longo, le médecin légiste. $\{S\}$ Déguisé comme les autres en fantôme a capuche, elle ne l'avait pas reconnu.

- Comment un tel homme avait-il pu devenir chef d'unité au CHS Pierre-Janet ? \{S\}Se pouvait-il qu'il soit le meurtrier de Philippe Duruy ? \{S\}Anaïs releva la tête. $\{S\}$ Elle sentait quelque chose ici. $\{S\}$ Elle examina les objets, les documents, les dossiers sur son bureau. $\{S\}$ Rien n'était dérangé mais chaque détail portait la trace d'un passage, d'une présence étrangère.

- Avant de refermer une dernière fois le corps dans son cercueil temporaire, son attention fut attirée par une légère tâche bleutée au niveau de la pomme d'Adam. Elle examina la marque après avoir pris le temps d'enfiler soigneusement un gant en caoutchouc. $\{S\}$ Un coup de poing, voire même une manipulation un peu sèche du médecin légiste auraient pu en être la cause.\{S\} Eve Saint Hilaire, méticuleuse et consciencieuse, décida de pratiquer une ouverture de la gorge pour éliminer toutes les hypothèses possibles.\{S\} En l'absence de scalpel et pour ne pas avoir à retourner dans son bureau, elle décida d'opérer avec le couteau qui était en sa possession.

- Au bout d'une heure, Holmes parvint a un cercueil en plomb qui se trouvait devant l'entrée de la voute.\{S\} J'entendis son petit cri de satisfaction et je vis a ses gestes hâtifs mais précis qu'il était arrivé au but.\{S\} Avec sa loupe, il examina soigneusement les bords du lourd couvercle. $\{S\}$ Puis il tira de sa poche une sorte de petite pince- monseigneur qu'il glissa dans un interstice, et il entreprit de soulever tout le devant, qui semblait n'être attaché que par deux crampons.

- Quand il rentra dans la chambre, Sartine s'était à nouveau affalé dans son fauteuil et paraissait en proie à un débat intérieur intense. $\{S\}$ Sans troubler sa réflexion, Nicolas revint vers le corps. \{S\}Le bougeoir à la main, il examina les lieux en commençant par le parquet. $\{S\}$ Il repéra quelques rayures de fraîche date, dont l'origine pouvait tout aussi bien provenir du gravier coincé sous la semelle des bottes que de toute autre cause. \{S\}Le 
dessus du bureau attira ensuite son attention. \{S\} Sous la lampe bouillotte placée au milieu du maroquin, il trouva une feuille de papier et il lut, écrit d'une main hâtive en grosses capitales, les mots «PARDON, ADIEU».

- Nous sommes deux, lui et moi, à avoir la clef et le secret... » Mais, pour ouvrir le coffre, il fallait enlever le corps et on attendit que la tâche des photographes fût terminée. $\{S\}$ Le médecin légiste faisait son rapport verbal. $\{S\}$ Couchet avait été atteint d'une balle dans la poitrine et l'aorte ayant été sectionnée, la mort avait été foudroyante. $\{S\}$ La distance entre l'assassin et sa victime pouvait être évaluée à trois mètres.\{S\} Enfin, la balle était du calibre le plus courant : 6,35 $\mathrm{mm}$.

\begin{tabular}{|l|l|l|l|l|}
\hline $\begin{array}{l}\text { N }<\text { HUM : POLICE }> \\
\text { les policiers }\end{array}$ & dessiner & contour & $\begin{array}{l}\text { du cadavre } \\
\varnothing\end{array}$ & $\begin{array}{l}\text { à la craie } \\
\text { avec de la craie }\end{array}$ \\
\hline
\end{tabular}

Tableau 1 : État des lieux 1.

\begin{tabular}{|l|l|l|l|}
\hline $\begin{array}{l}\text { N }<\text { HUM : POLICE }> \\
\text { les policiers } \\
\text { les photographes }\end{array}$ & photo & $\begin{array}{l}\text { du cadavre } \\
\text { du corps } \\
\varnothing\end{array}$ \\
\hline
\end{tabular}

Tableau 2 : État des lieux 2.

\begin{tabular}{|c|c|c|c|}
\hline $\begin{array}{l}N<H U M \text { : POLICE }> \\
\text { les policiers } \\
\text { le commissaire }\end{array}$ & examiner & $\begin{array}{l}\text { soigneusement } \\
\text { le nez au sol }\end{array}$ & $\begin{array}{l}N<\text { lieux }> \\
\text { les lieux } \\
\text { la zone qui l'intéressait } \\
\text { le parquet } \\
\text { le sol } \\
\text { la pièce } \\
N<\text { INC }> \\
\text { objets }\{M O D I F\} \\
\text { documents }\{M O D I F\} \\
\text { dossiers }\{M O D I F\}\end{array}$ \\
\hline $\begin{array}{l}N<H U M \text { : POLICE }> \\
\text { le médecin } \\
\text { le médecin légiste }\end{array}$ & & & $\begin{array}{l}N<H U M \_M O R T> \\
\text { cadavre } \\
\text { corps }\end{array}$ \\
\hline
\end{tabular}

Tableau 3 : État des lieux 3.

\begin{tabular}{|l|l|l|}
\hline $\begin{array}{l}N<H U M \\
\text { médecin légiste }\end{array}$ & $\begin{array}{l}\text { premières conclusions } \\
\text { avis } \\
\text { rapport verbal } \\
\text { livrer } \\
\text { faire }\end{array}$ & \\
\hline
\end{tabular}

Tableau 4 : État des lieux 4.

\subsection{Collecte des traces, indices et preuves}

a) Si bien qu'à huit heures du matin au plus tard quelqu'un connaissait la disparition de Jean Servières, savait que l'auto était ou serait abandonnée près de la rivière Saint-Jacques et qu'on relèverait des traces de sang sur le siège... Et ce quelqu'un, par surcroît, n'ignorait pas que l'on découvrirait quelque part les empreintes d'un inconnu aux grands pieds...-C'est incroyable !... soupira l'inspecteur. \{S\} Quant à ces empreintes, je les ai expédiées au Quai des Orfèvres par bélinogramme.\{S\} Ils ont déjà consulté les sommiers. \{S\} J'ai la réponse : 
elles ne correspondent à aucune fiche de malfaiteur...Il n'y avait pas à s'y tromper : Leroy se laissait gagner par la peur ambiante.

b) La pièce est un peu humide, ils ont dû laisser une trace de rouille ou une petite empreinte.

c) Ne t'a-t-elle pas laissé quelques indices qui puissent nous aider?

d) L'assassin ne laisse aucun indice.

e) Il y a des traces de l'assassin partout... jeune homme

\begin{tabular}{|c|c|c|c|c|c|c|c|}
\hline $\begin{array}{l}N<H U M \text { : POLICE }> \\
\text { policiers } \\
\text { médecin } \\
\text { médecin légiste }\end{array}$ & \multirow[t]{3}{*}{$\begin{array}{l}\varnothing \\
\text { ne pas } \\
\text { ne } \\
\text { aucun }\end{array}$} & $\begin{array}{l}\text { Relever } \\
\text { découvrir }\end{array}$ & \multirow[t]{3}{*}{$\begin{array}{l}\text { trace } \\
\text { indice } \\
\text { empreinte }\end{array}$} & \multirow{3}{*}{$\begin{array}{l}\varnothing \\
\text { de pieds } \\
\text { de doigts } \\
\text { de sang } \\
\text { de rouille } \\
\text { de son } \\
\text { passage }\end{array}$} & $\begin{array}{l}\text { de } \\
\text { l'assassin }\end{array}$ & & \multirow[t]{3}{*}{$\begin{array}{l}<L O C> \\
\text { sur le siège } \\
\text { quelque part } \\
\text { partout }\end{array}$} \\
\hline \multirow[t]{2}{*}{$\begin{array}{l}N<H U M \\
\text { COUPABLE }> \\
\text { assassin } \\
\text { coupable } \\
\text { meurtrier }\end{array}$} & & laisser & & & & $\begin{array}{l}\text { après } \\
\text { lui/elle } \\
\text { derrière } \\
\text { lui/elle }\end{array}$ & \\
\hline & & Il y a & & & $\begin{array}{l}\text { de } \\
\text { l'assassin }\end{array}$ & & \\
\hline
\end{tabular}

Tableau 5 : Collecte des traces.

\subsection{Description du corps}

a) Le corps gisait dans une position impossible.

b) Nicolas eut la vision fugitive d'un corps gisant dans la neige et d'un cadavre sur la table de la basse-geôle. ... en position visible dans l'atelier de M. Le Roy et cela pour prendre l'ennemi sur le temps.

c) Elle avait l'impression qu'on avait substitué un zombie à son frère chéri.\{S\} Ce gisant allongé dans le salon.

d) C'est là, dans ce passage très mal éclairé, qu'il a été découvert gisant par terre sans connaissance. _ Qui l'a découvert? _ Un homme de service.

e) Un corps désarticulé gisait à même le sol.\{S\} Une multitude de rats affolés quittèrent avec précipitation les viscères du cadavre ensanglanté.

\begin{tabular}{|c|c|c|c|c|c|}
\hline $\begin{array}{l}N<H U M> \\
\text { corps } \\
\text { corps de } \\
N<\text { hum }> \\
\text { cadavre* }\end{array}$ & $\begin{array}{l}\text { désarticulé } \\
\text { ligoté }\end{array}$ & $\begin{array}{l}\text { gésir } \\
\text { gisant }\end{array}$ & $\begin{array}{l}<\text { POSITION }> \\
\text { dans une position } \\
\text { impossible } \\
\text { allongé }\end{array}$ & $\begin{array}{l}<M A N I E ̀ R E> \\
\text { sans } \\
\text { connaissance* }\end{array}$ & $\begin{array}{l}<L O C> \\
\text { dans la neige } \\
\text { sur une plage de la } \\
\text { côte ouest } \\
\text { dans la calanque de } \\
\text { Sormiou } \\
\text { dans le salon }\end{array}$ \\
\hline
\end{tabular}




\begin{tabular}{|l|l|l|l|l|}
\hline & & & & $\begin{array}{l}\text { chez vous } \\
\text { dans les sous-sols de } \\
\text { sa chère école } \\
\text { sur la table } \\
\text { sur le tapis } \\
\text { par terre } \\
\end{array}$
\end{tabular}

Tableau 6 : Description du corps 1.

f) Je crains que tu te méprennes sur mes paroles : je l'ai retrouvée, morte ! -Morte ? - Plus précisément assassinée, d'une balle dans la tête. $\{S\}$ Le corps a été découvert par une patrouille, gisant dans les douves des Invalides, côté ville. On les a retrouvés morts, empoisonnés.

g) Ou au pyromane retrouvé mort d'une hémorragie interne, le matin même, après s'être fait sodomiser par son doberman.

h) Vous parlez d'une fille retrouvée morte sur une plage de la côte ouest, répondit Osborne.

\begin{tabular}{|c|c|c|c|c|c|c|}
\hline $\begin{array}{l}N<H U M> \\
\text { on }\end{array}$ & $\begin{array}{l}N 1<H U M> \\
\text { PRON } \\
\text { PERSONNEL }\end{array}$ & $\begin{array}{l}\text { retrouver } \\
\text { être } \\
\text { retrouvé }\end{array}$ & mort & $\begin{array}{l}\text { CAUSE } \\
\text { assassinée, d'une } \\
\text { balle dans la tête, } \\
\text { empoisonné, } \\
\text { d'une hémorragie } \\
\text { interne, } \\
\text { pendu }\end{array}$ & $\begin{array}{l}<L O C> \\
\text { Sur une plage } \\
\text { de la côte ouest }\end{array}$ & $\begin{array}{l}<\text { TEMPS }> \\
\text { le } \\
\text { décembre } \\
\text { 2009, } \\
\text { hier, } \\
\text { hier soir, } \\
\text { le matin même }\end{array}$ \\
\hline
\end{tabular}

Tableau 7 : Description du corps 2.

i) Avec stupeur, il avait découvert le corps ligoté de son officier transpercé de multiples coups de couteau

j) Lorsque j’ai découvert le corps de mon ami, et celui de Sophie, je me suis évanoui.

k) Cela s'est produit dans la nuit de samedi à dimanche, on a découvert le corps hier matin.\{S\} L'alerte a été donnée par son jardinier, rentré vers cinq heures.

l) On a découvert son corps au fond du puits. $\{S\}$

\begin{tabular}{|l|l|l|l|l|l|}
\hline on $<$ HUM $>$ & découvrir & $\begin{array}{l}\text { Corps } \\
\text { Corps de } \\
N<\text { hum }>\end{array}$ & désarticulé, & ligoté, \\
& & $\begin{array}{l}\text { transpercé de multiples } \\
\text { coups de couteau, }\end{array}$ & $\begin{array}{l}\text { au fond du } \\
\text { puits }\end{array}$ & $\begin{array}{l}\text { le 17 décembre } \\
2009, \\
\text { hier, }\end{array}$ \\
& & & & hier soir, \\
\hline
\end{tabular}




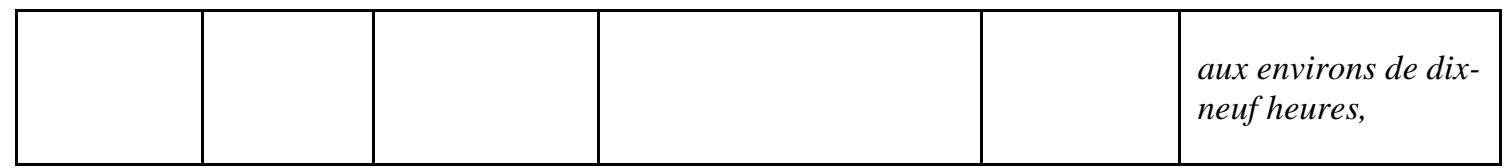

Tableau 8 : Description du corps 3.

m) Aux environs de dix-neuf heures, hier, le 12 novembre 1988, le corps de Manon Simonis, huit ans, a été découvert au fond d'un puits de dispersion, près de la station d'épuration de la ville.

n) Le corps d'une femme nue a été découvert hier matin, à quelques kilomètres de Sartuis (haut Doubs), dans le parc naturel de la fondation de Notre-Dame-de-Bienfaisance.

\begin{tabular}{|c|c|c|c|c|c|c|}
\hline $\begin{array}{l}\text { Corps } \\
\text { Corps de } \\
N<\text { hum }>\end{array}$ & $\begin{array}{l}\text { désarticulé, } \\
\text { ligoté, } \\
\text { transpercé de } \\
\text { multiples } \\
\text { coups de } \\
\text { couteau, }\end{array}$ & $\begin{array}{l}\text { être } \\
\text { découvert }\end{array}$ & par & $N<H U M>$ & $\begin{array}{l}<L O C> \\
\text { au fond d'un puits de } \\
\text { dispersion, près de la } \\
\text { station d'épuration de la } \\
\text { ville. } \\
\text { à quelques kilomètres de } \\
\text { Sartuis (haut Doubs), } \\
\text { dans le parc naturel de } \\
\text { la fondation de Notre- } \\
\text { Dame-de-Bienfaisance.. }\end{array}$ & $\begin{array}{l}<T E M P S> \\
\text { le } 17 \\
\text { décembre } \\
2009, \\
\text { hier, } \\
\text { hier soir, } \\
\text { aux environs } \\
\text { de dix-neuf } \\
\text { heures }\end{array}$ \\
\hline
\end{tabular}

Tableau 9 : Description du corps 4.

L'analyse de corpus nous a permis de regrouper les structures RLS propres à la scène de crime sous forme des tableaux comme ceux présentés ci-dessus[8]. Les constructions dégagées, ayant la valeur de constructions préfabriquées, peuvent commuter, comme nous l'avons mentionné dans le chapitre précédent, et, finalement, être réutilisées pour proposer de nouvelles scènes de crime. Nous le démontrons ci-dessous en modifiant un fragment du texte authentique de Bastiani.

\section{Texte original :}

- Putain, c’est pas vrai ! lâcha Esposito

- On est dans la merde, confirma Lepage.

Ils regardaient sans trop y croire le cadavre de Grangier, le directeur de l'ESCOM en personne, retrouvé mort dans les sous-sols de sa chère école. À genoux contre un mur, les mains liées dans le dos. La gorge tranchée. Le médecin légiste livra ses premières conclusions.

— Il a été tué il y a moins de deux heures, annonça-t-il.

— Putain, on est dans la merde ! répéta Lepage. C’était pas Zamikellian. Ce fou s’est bien foutu de nous!

Esposito restait pétrifié face au corps sans vie. Tout s'écroulait, il ne comprenait plus.

Et, soudain, son cerveau se remit à fonctionner.

- Merde ! s’écria-t-il.

\section{Textes modifiés :}


Ils regardaient sans trop y croire le cadavre de $\mathrm{X}, \mathrm{X}$ retrouvé mort dans la cave de sa maison. Par terre, le corps contorsionne. le visage trouble, aux couleurs violentes, les yeux exorbités. Le médecin légiste faisait son rapport verbal. La victime a été empoisonnée, il y a moins de trois heures, [...].

Ils regardaient sans trop y croire le corps de $\mathrm{X},[\ldots]$, découvert dans le salon de sa maison. À genoux contre un mur, ligoté, transpercé de multiples coups de couteau. Le médecin légiste livra ses premières conclusions. La mort remontait à deux jours, minimum $[\ldots]$.

\section{Conclusion}

La présente analyse est une des étapes qui vont nous permettre de décrire l’organisation linguistique / langagière du roman policier. Quels sont les motifs sémantiques et les structures rhétorico-lexicosyntaxiques normatifs qui nous autorisent à classer un texte comme roman policier, si l'on sait, par exemple, qu'il existe des textes qui contiennent des scènes de crime et qui ne peuvent pas être considérés comme représentants du genre?

Afin d'obtenir une image linguistique précise du genre, il faut prendre en compte quelques postulats méthodologiques qui « clarifieraient » l'analyse, par exemple :

- $\quad$ créer des corpus formés uniquement de représentants du genre généralement reconnus comme tels ;

- $\quad$ accepter qu'un genre possède une structure interne multiple et mobile ;

- $\quad$ accepter que la structure en question contienne des éléments obligatoires et facultatifs, etc.

Un de nos objectifs est de mettre à la disposition de la communauté scientifique une interface de consultation permettant d'effectuer des recherches personnelles, mais avant tout des recherches structurées autour du roman policier. Le CRIMOLEX mettra à la disposition des chercheurs un large corpus de romans policiers. Il aura une double compétence, (i) en tant que concordancier, il permettra d'effectuer des recherches des mots dans un roman policier, mais avant tout il permettra de (ii) retracer automatiquement des structures (paramétrées selon les règles lexico-syntaxiques) caractéristiques pour le roman policier. Cet outil pourra répondre aux besoins des linguistes, des étudiants, des traducteurs (il s’agit de corpus comparables français-polonais), des écrivains et de tous ceux qui s’intéressent au roman policier.

Cette étude semble pertinente et aisée pour un genre très codé, comme le roman policier. La question de son applicabilité à des corpus moins contraints ou prévisibles n'est pas posée car elle dépasse le cadre de la présente analyse. À l'étape actuelle de nos recherches, il est trop tôt pour projeter des résultats obtenus sur un autre genre textuel.

\section{Références bibliographiques}

Barthes, R. (1966). « Introduction à l'analyse structurale des récits ». Communications, 8, Recherches sémiologiques : l'analyse structurale du récit, p. 1-27.

Barthes, R. (1964). «Structure du fait divers », Essais critiques, Paris : Seuil.

Beauvisage, T. (2001). « Exploiter des données morphosyntaxiques pour l'étude statistique des genres application au roman policier ». Une version augmentée d'un article paru dans la revue T.A.L., $n^{\circ} 43$..

Le texte intégral disponible à http://www.revuetexto.net/Inedits/Beauvisage/index.html

Beliakov, V., Mejri, S. (dir.) (2015). Stéréotypie et figement. À l'origine du sens. Presses Universitaires du Midi.

Benveniste, E. (1976). Problèmes de linguistique générale, vol.1. Paris : Gallimard. 
Brecht, B. (1970). Les arts et la révolution, précédé de Notes sur le travail littéraire. Article sur la littérature. Paris : L'Arche.

Ducrot, O., Todorov, T. (1972). Dictionnaire encyclopédique des sciences du langage. Paris : Seuil.

Ehrlich, M.-F. (1994). Mémoire et compréhension du langage. Lille : P.U. de Lille.

François, M. (2009). « Le stéréotype dans le roman policier ». Cahiers de Narratologie, [En ligne], 17 | 2009, mis en ligne le 22 décembre 2009, consulté le 30 mars 2015. URL : http://narratologie.revues.org/1095.

Greimas, A. J. (éds.) (1982). Essais de sémiotique poétique. Paris : Larousse.

Grossmann, F., Tutin, A. (2003). Les collocations : analyse et traitement, Travaux et recherches en linguistique appliquée. Amsterdam : de Werelt.

Grossmann, F. (2015). « Les motifs du constat dans les genres scientifiques ». Stéréotypie et figement. À l'origine du sens, Beliakov, Vladimir, Mejri, Salah, (dir.) (2015). Presse Universitaire du Midi, p. 39-56.

Henry, J., http://polarophiles.lescigales.org/domaines.html

Kintsch, W. (1998). Comprehension: A paradigm for cognition, New York: Cambridge University Press.

Lasić, S. (1972). Poetyka powieści kryminalnej: próba analizy strukturalnej. Warszawa : Państwowy Instytut Wydawniczy.

Leech G., Short, M. (2007). Style in fiction: A linguistic introduction to english fictional prose. Harlow, Pearson.

Legallois, D., Tutin, A. (2013). «Vers une extension du domaine de la phraséologie ». Langages 189.

Legallois, D. (2006). « Des phrases entre elles à l'unité réticulaire du texte ». Langages, $n^{\circ}$ 163, p. 56-70.

Lits, M. (1993). Le roman policier: introduction à la théorie et à l'histoire d'un genre littéraire, Liège : Editions du C.E.F.A.L.

Longrée, D., Mellet, S. (2013). « Le motif : une unité englobante ? Étendre le champ de la phraséologie de la langue au discours ». Langages 189, p. 65-80.

López, A. C. ; Séré, A. (2001). La lectura en lengua extranjera. El caso de las lenguas románicas. Hamburg: Romanistik in Geschichte und Gegenwart, Beiheft 6. Buske

Magri-Mourgues, V. (2006). « Stylistique générique et statistique », Les Cahiers de la MSH Ledoux 8, p. 655-666.

Mejri, S. (2008). «Construction à verbes supports, collocations et locutions verbales ». Las construcciones verbonominales libres y fijas. Aproximación contrastiva y traductológica, Pedro Mogorron Huerta \& Salah, Mejri (éds), p. 191-202.

Narcejac, T. (1947). Esthétique du roman policier. Paris : Le Portulan.

Neveu, F. (2004). Dictionnaire des sciences du langage, Paris : Armand Colin.

Mejri, S., Muryn, T., et al. (éds). (2013). La phraséologie entre langues et cultures. Structures fonctionnement, discours, Peter Lang.

Mejri, S., Muryn, T. (éds) (2015). Linguistique du discours: de l'intra- à l'interphrastique, Peter Lang.

Minsky, M. (1975). A Framework for Representing Knowledge. The Psychology of Computer Vision, P. H. Winston (ed.), McGraw-Hill.

Todorov, T. (1971). « Typologie du roman policier ». Poétique de la prose, Paris : Seuil.

Rastier, F., Malrieu, D. (2000). Discours, genres et typologie de textes.

Riegel, M., Pellat, J.-Ch., Rioul, R. (1994). Grammaire méthodique du français, Paris : PUF.

Schank, R. C., Abelson, R. (1977). Scripts, plans, goals, and understanding: An Inquiry into human knowledge structures. Hillsdale, N.J. New York: L. Erlbaum Associates distributed by the Halsted Press Division of J. Wiley and Sons.

Sinclair, J. McH. (2004). Trust the text: Language, corpus and discourse, London : Routledge.

Sketchengine. http://www.sketchengine.co.uk. 
Stubbs, M. (2005). « Conrad in the computer: examples of quantitative stylistic methods ». Language and Literature, 14 (1), p. 5-24.

Tutin, A., Grossmann, F. (2002). « Collocations régulières et irrégulières : esquisse de typologie du phénomène collocatif ». Revue française de linguistique appliquée, 2002/1 (Vol. VII), p. 7-25.

Van Dijk, T. A. (2008). Discourse and Context. À sociocognitive approach. Cambridge: Cambridge University Press.

Van Oostendorp, H., Goldman, S. R. (eds) (1999). The construction of mental representations during reading, Mahwah, NJ: Lawrence Erlbaum.

[1] Cependant, il faut évoquer des études en stylistique (Barthes, 1964, 1966 ; Greimas, 1982 ; Leech \& Short, 1981), en stylométrie (Magri-Mourgues, 2006), en stylistique de corpus (Stubbs, 2005 ; Beauvisage, 2001) qui traitent des procédés de style et des spécificités lexicales et grammaticales chez différents auteurs ou qui établissent des modelés stéréotypiques sous-jacents, p. ex. pour le roman policier (Todorov, 1980 ; François, 2009 ; Lits, 2011).

[2] La notion de matrice utilisée dans cette recherche sera définie dans la suite de l'article

[3] Le texte intégral disponible sur http://www.revue-texto.net/Inedits/Beauvisage/index.html)

[4] Le texte intégral disponible sur http://www.revue-texto.net/Inedits/Beauvisage/index.html

[5] Les citations utilisées dans l'article ne sont pas sourcées puisque l'une de nos hypothèses de départ est l'existence d'une matrice stable. Aussi, la provenance des textes n'a-t-elle pas tant d'importance, une fois que nous savons que ceux-ci appartiennent au genre policier et que le corpus d'analyse est équilibré (chaque auteur étant représenté par un nombre comparable de romans).

[6] Malgré l'intérêt des linguistes pour la description morphosyntaxique et syntaxico-sémantique de séquences libres et de séquences figées, les collocations sont restées à l'écart du champ d'investigation. Il $\mathrm{y}$ a très peu de travaux concentrés particulièrement sur ce sujet (P. Blumenthal, F. Grossman, F. J. Haussman, I. Mel'čuk, S. Mejri, A. Polguère, D. Siepmann, A. Tutin, G. Gréciano - pour le français et J. S. Bień, K. Szafran (les travaux d'A. Buczyński et de T. Okniński) - pour le polonais.

[7] Nous postulons l'existence des éléments normatifs appartenant à plusieurs niveaux d'analyses linguistiques, mais obligatoires pour qu'un texte soit défini comme représentatif pour un genre.

[9] Cependant, nous tenons à préciser qu'il s'agit d'un travail de longue haleine et qui demandera encore des discussions et des réflexions. 